\title{
Evaluation of the Training and Pilot Implementation of Katatagan Kontra Droga sa Komunidad
}

\author{
Ma. Regina M. Hechanova \\ Arsenio S. Alianan \\ Mendiola T. Calleja \\ Avegale C. Acosta \\ Camille C. Yusay \\ Ateneo de Manila University
}

\begin{abstract}
This study evaluated the training of facilitators and pilot implementation of the Katatagan Kontra Droga sa Komunidad (KKDK), a communitybased drug recovery program. Paired samples t-test of pre and posttest scores of 111 community facilitators who underwent training revealed significant changes in their perceived competence, motivation, and commitment. The program was pilot-tested among 46 mild-risk drug users. Pre and posttest results revealed moderate effects in substance use dependence symptoms and life skills, and large effects in drug recovery skills and psychological well-being. Correlational analysis of posttest scores revealed a negative relation between life skills and SUD symptoms and a positive relation between recovery skills and psychological well-being. Post-program focus group discussions with participants and interviews with facilitators highlighted the value of building recovery and life skills in enabling change in the participants and their families. However, field observations revealed a number of enablers and challenges in implementation.
\end{abstract}

Keywords: substance use, drug prevention, drug recovery, communitybased interventions

In July 2016, the government launched its campaign against illegal drugs dubbed as Oplan Tokhang. As part of this, community officials and the local police went to the homes of known drug users and asked

Correspondence concerning this article can be addressed to Ma. Regina M. Hechanova, PhD, Department of Psychology, Leong Hall, Ateneo de Manila University, Katipunan Avenue, Loyola Heights 1108 Quezon City Philippines. Email: rhechanova@ateneo.edu 
them to voluntarily surrender (Sadongdong, 2018). The Dangerous Drugs Board of the Philippines (DDB) declared that majority of those who surrendered were low- to mild-risk users who could undergo rehabilitation in their communities (Cepeda, 2016). Unfortunately, drug treatment in the Philippines has typically been conducted in inpatient rehabilitation centers or through the criminal justice system, and the country does not have a robust tradition of communitybased drug recovery (CBDR). In response to this, the Psychological Association of the Philippines (PAP) created the Katatagan Kontra Droga sa Komunidad or KKDK (Resilience Against Drugs in the Community), an evidence-informed and culturally-adapted treatment program for mild-risk drug users (Hechanova, Alianan, et al., 2018). This paper evaluates the training of community facilitators and the pilot implementation of KKDK. Given the dearth of literature on substance use recovery and treatment in the Philippines, it contributes to both knowledge and practice by highlighting initial outcomes as well as challenges in implementing community-based drug treatment.

\section{Theories and Treatment of Drug Use}

Drug use has been viewed in many ways. The moral perspective views drug use as a sin and drug dependents as morally weak. The enlightenment perspective, on the other hand, views drug use as a result of an existential vacuum or spiritual void (Skewes \& Gonzales, 2013). Alternatively, the medical model suggests that drug use has biological predispositions such as comorbid disorders and genetic predispositions. Psychological theories present substance use as a problematic behavior driven by negative emotions, faulty cognitions, and/or their interaction (Skewes \& Gonzales, 2013). In addition, a number of socioecological theories suggest that drug use is socially learned through the family, environment (Stanton, 1980) or influenced by culture (Lukoff, 1980).

However, today, the most current perspective brings together these different models and suggests that drug use as a multifaceted health problem with biological, psychological, personality, cognitive, social, cultural, and environmental roots. Given this, treatment for drug use has also become increasingly multifaceted and there is 
evidence that a combination of treatments is superior to just one type of treatment. Skewes and Gonzalez's (2013) review of drug treatments reported that the most successful treatment programs incorporate strategies that enhance individuals' ability to reduce cravings, manage triggers, and prevent relapse. In addition, the ability to manage difficult emotions, cope with negative life circumstances, promote social support for sobriety, and establish a healthy lifestyle are critical to long-term recovery.

\section{Community-based Drug Treatment}

The view of drug use as a complex issue has also shifted the management of drug use from a punitive to a reformative perspective. The United Nations Office on Drugs and Crime (UNODC; 2014) advocates that people who use drugs (PWUDs) should be treated in the health care system using a holistic biopsychosocial approach rather than through the criminal justice system. Because institutionalized drug treatment programs are expensive and often inaccessible to the poor, greater attention has been given to the role of community-based drug treatment and recovery support.

Community-based Drug Recovery (CBDR) support involves providing holistic care ranging from prevention and health promotion, screening and assessment, treatment and rehabilitation, education, skills training, livelihood opportunities, and other relevant social services closest to where the people are. Compared to inpatient programs, community-based interventions are less disruptive because recovering users are not taken away from their sources of support and livelihood while they are seeking treatment (UNODC, 2014). In fact, there is evidence that implementing community-based interventions are not only less costly, they also bring about significant decreases in emergency room visits, hospital stay, and criminality (UNODC, 2014).

However, not all community-based interventions are equally efficacious. The UNODC (2014) guidelines for CBDR advocates the importance of using evidence-based treatments that are appropriate to the culture of clients. There is evidence that culturally-adapted substance use interventions are more effective than non-adapted interventions in Latin America (Robles, Maynard, Salas-Wright, \& 
Todic, 2018) and among racial minorities (Hodge, Jackson, \& Vaugh, 2012).

\section{Development of Katatagan Kontra Droga sa Komunidad}

To respond to the gap in evidence-informed drug interventions, the Psychological Association of the Philippines Special Interest Group on Substance Use Prevention and Recovery developed the Katatagan Kontra Droga sa Komunidad (KKDK; Resilience Against Drugs in the Community). The KKDK intervention was developed using McKleroy and colleagues' (2006) design process that consisted of five stages: (a) assessment of needs, (b) development of intervention, (c) training of facilitators, (d) pilot-testing, and (e) implementation (Hechanova, Alianan, et al., 2018). In addition, to ensure the program is culturally appropriate and relevant, the design team used a community-based, participatory action research approach (Collins et al., 2018).

A needs analysis was conducted with PWUDs from a community in Metro Manila. The interviews highlighted the biopsychosocial nature of drug use. In terms of the biological factors, majority of interviewees reported using shabu (methamphetamine) and marijuana with some beginning as early as 11 years old. Some PWUDs also reported drug use in the family (Hechanova, Alianan, et al., 2018).

The interviews also highlighted the psychological dimensions of drug use. Some persons who use drugs (PWUDs) shared that they used drugs for recreational purposes and to feel good. However, there were also those who used drugs for functional purposes-to assuage hunger or to have more energy to work longer and harder. Many PWUDs also cited drug use was a way of escaping adverse childhood experiences or coping with multiple life problems.

Despite voluntarily surrendering, some PWUDS appeared ambivalent about stopping. For some, this was because they had not yet experienced any negative effects of their drug use. However, their ambivalence may also be attributed to a lack of self-efficacy. Half of PWUDs reported that they have tried to stop in the past but have lapsed. Interviewers also noted the lack of drug recovery and life skills (Hechanova, Alianan, et al., 2018).

In terms of social factors, the interviews highlighted the salience 
of poverty and its vulnerabilities. Majority of participants were poor, uneducated, and unemployed. Two-thirds of interviewees grew up in violent communities and experienced parental physical and emotional abuse or neglect. The interviews also highlighted the influence of peer and family use as a reason for their initial use. At the same time, majority of interviewees also identified their families as their motivation to change (Hechanova, Alianan, et al., 2018).

Given the results of the needs analysis, the research team examined the Substance Abuse and Mental Health Services Administration (SAMHSA) Matrix Intensive Outpatient (MIOP) modules (2006), that was endorsed by the Department of Health (DOH) for moderate risk users, and the UNODC Trainer's Manual for Community-Based Drug Recovery Support (UNODC, 2015). The contents of these modules were matched with the psychosocial needs of PWUDs and a program framework was developed consisting of drug recovery skills, life skills, and family modules (see Figure 1).

Using a community participatory approach, the initial program framework was validated with stakeholders consisting of local government officials, church workers, antidrug abuse personnel, addiction professionals, health professionals, and recovering users. Community stakeholders affirmed the importance of providing drug recovery and life skills to clients but raised concerns about the suitability of the UNODC and MIOP modules given participants' low literacy levels and the lack of resources. They suggested the need to make the modules more interactive and culturally-appropriate.

The resulting intervention was entitled Katatagan Kontra Droga sa Komunidad (KKDK) (Resilience Against Drugs in the Community). It utilizes an integrative approach blending a number of treatments that have robust evidence of efficacy in drug treatment: motivational interviewing, cognitive behavioral therapy, mindfulness, and family systems theory (Psychological Association of the Philippines [PAP], 2017). A summary of the program is found in Table 1 but a more indepth description is found in Hechanova, Alianan, et al. (2018).

Motivational interviewing (MI) is a client-focused approach used in treatment of substance use and other behavioral problems. It assumes that people will not change simply because they are told to do so. It aims to elicit users' internal motivation to change through the use of 


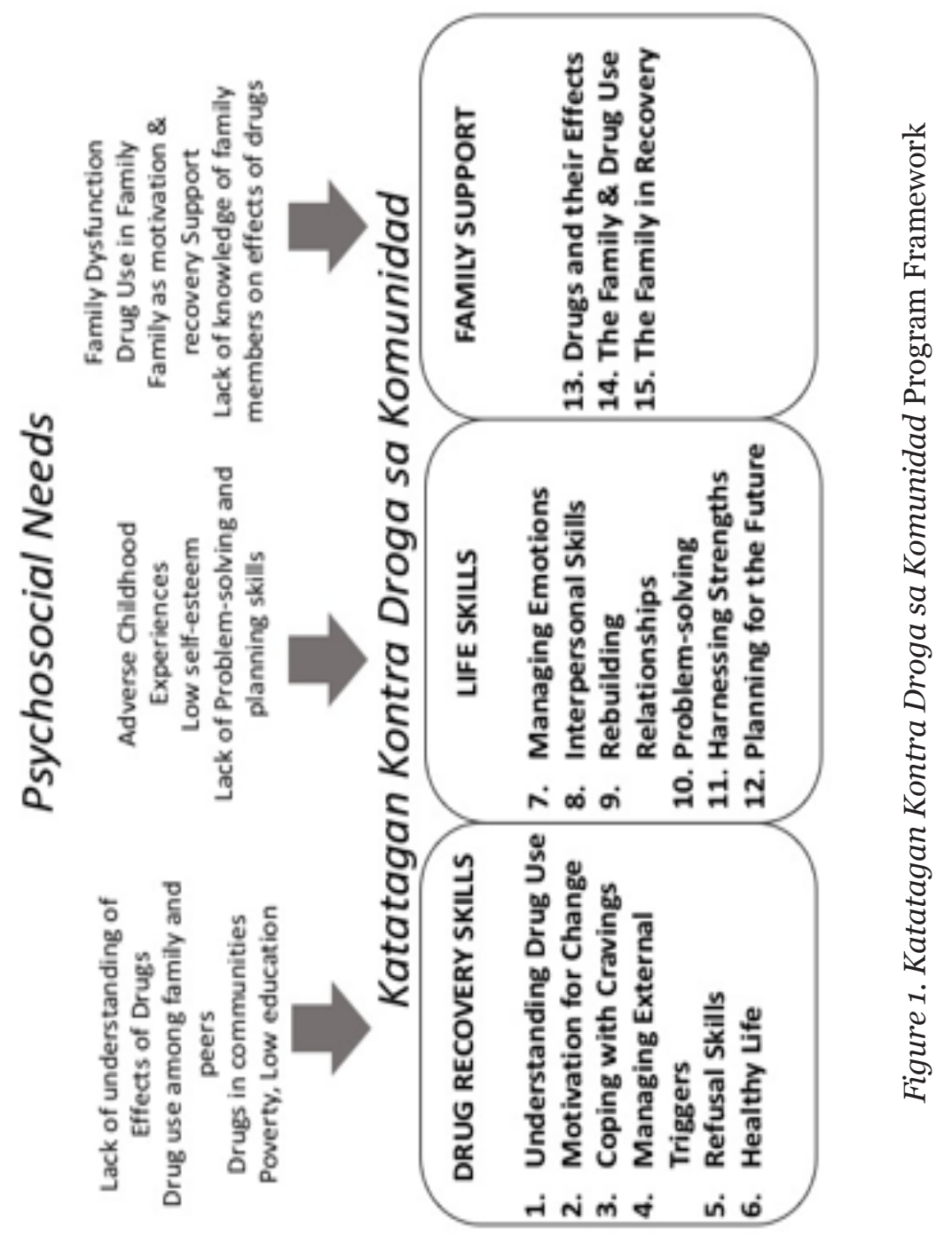




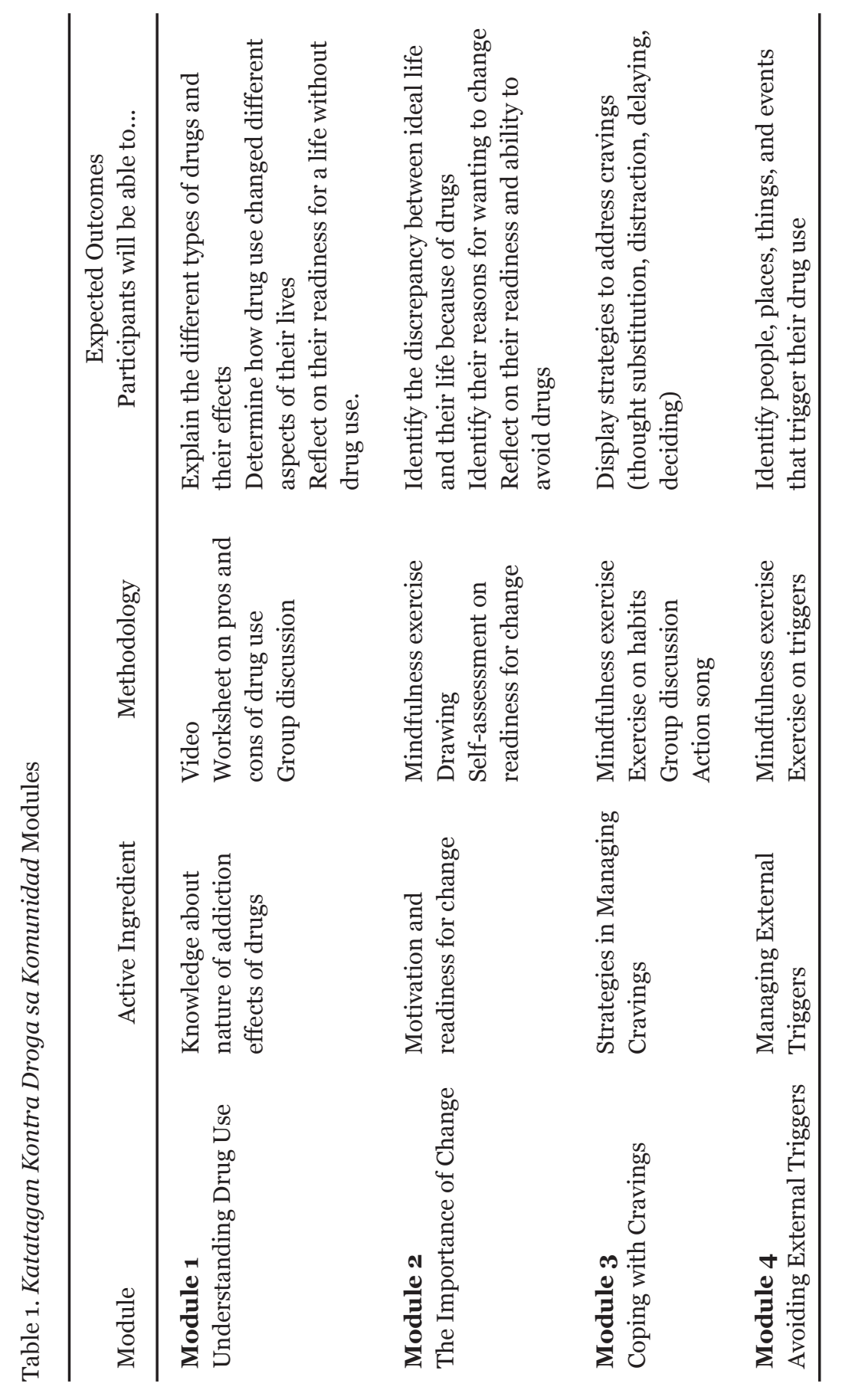




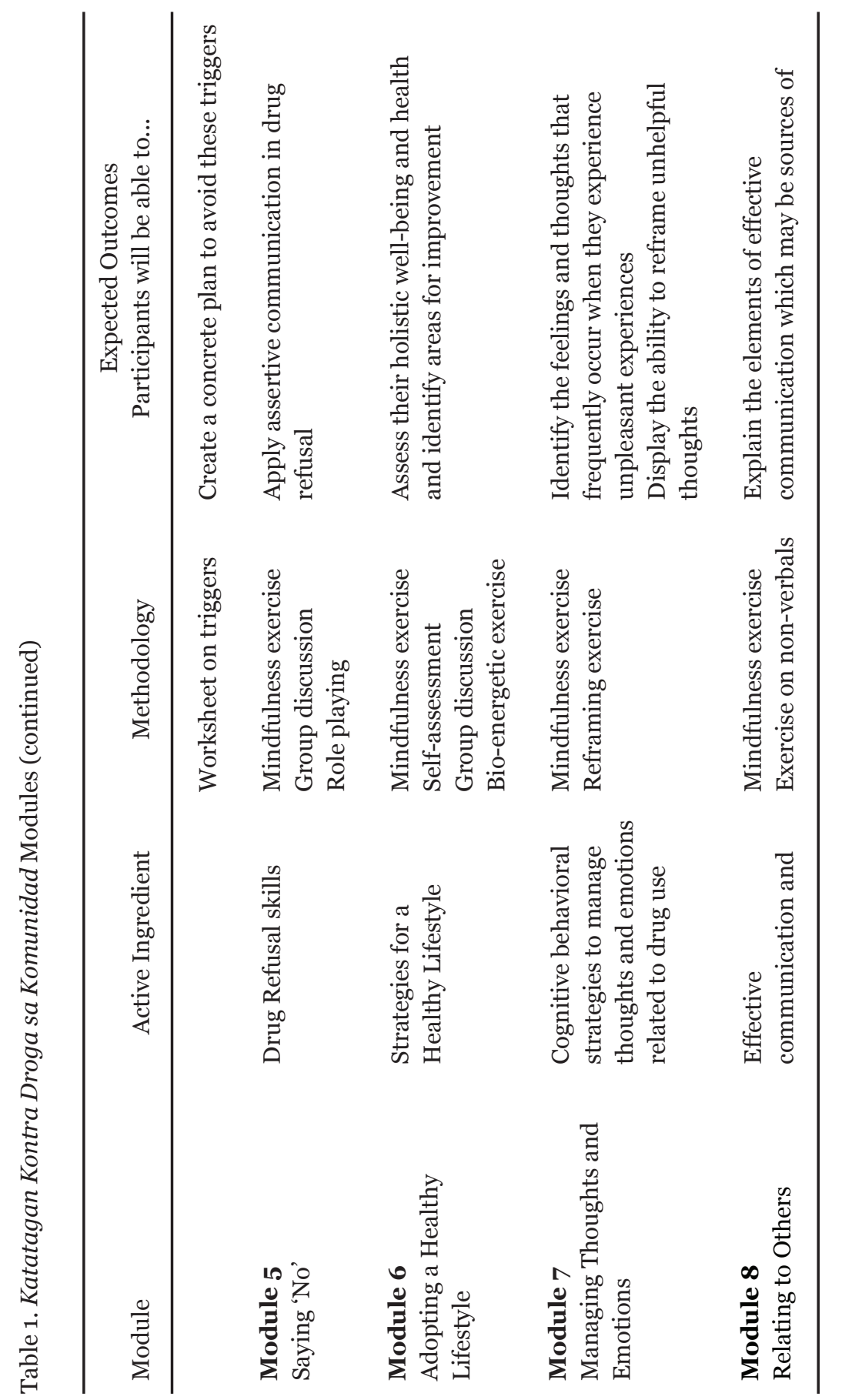




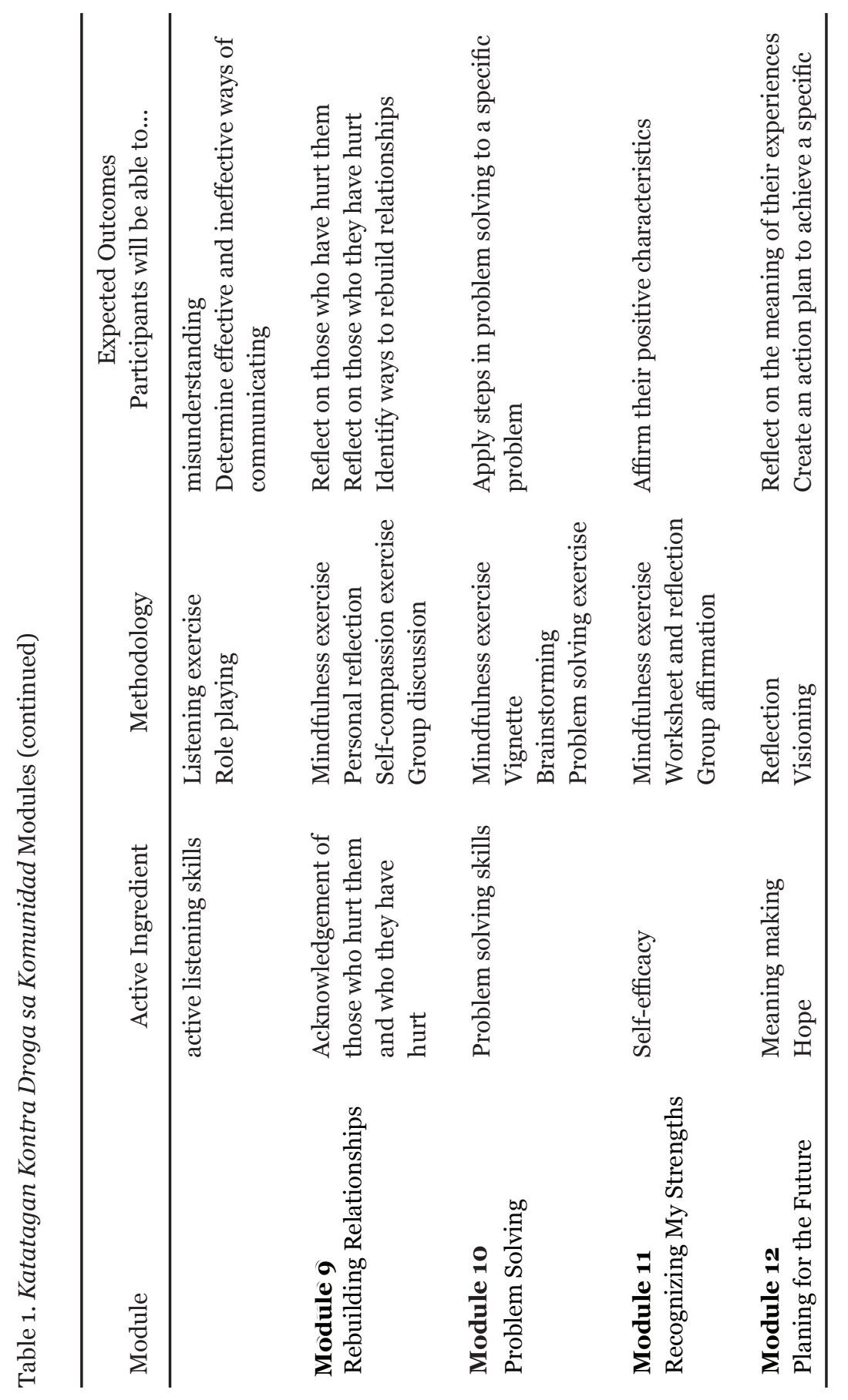




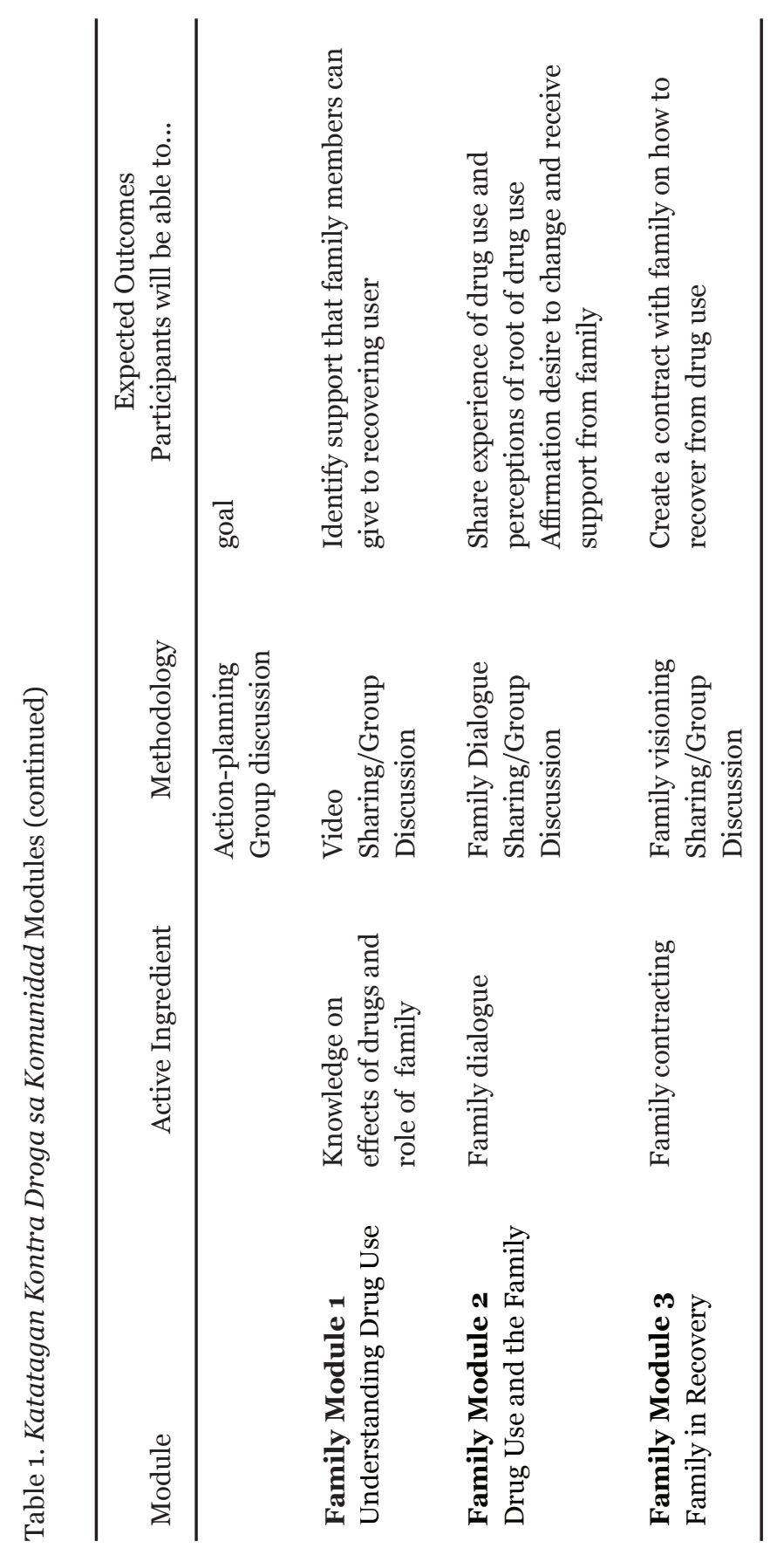


empathy, increasing discrepancy between ideal and current situation, and fostering a sense of self-efficacy and optimism (Miller \& Rollnick, 2012). The KKDK training for community facilitators includes MI skills such as using open questions, affirmation, reflective listening, rolling with resistance, etc. MI principles are also used in the design of the modules. The first two modules of KKDK focus on increasing PWUDs motivation to change. In Module 1, PWUDs reflect on the positive and negative effects of their drug use. In Module 2, participants envision their ideal life and compare this with their current life to highlight discrepancy and assess their readiness to change. The goal of MI to encourage self-efficacy is also evident in Module 11 where participants are asked to reflect on their strengths and how they have changed. To improve commitment to change participants are encouraged to plan for a better future in Module 12 (PAP, 2017).

The KKDK program also uses principles of cognitive behavior therapy (CBT) that has robust evidence in the treatment of substance use (Windsor, Jemal, \& Alessi, 2015). Beck, Wright, Newman, and Liese (1993) suggest drug use is a learned behavior that can be unlearned by addressing seven areas of psychological vulnerability: 1) high risk situations that are both external (People, places, things) and internal (mood states), 2) dysfunctional beliefs about drugs, oneself and one's relationship with drugs, 3) automatic thoughts that increase arousal and intent to use, 4) physiological cravings, 5) permission-giving beliefs that justify drug use, 6) rituals and behaviors linked to substance use and 7) adverse reactions to lapse or relapse. CBT can thus help recovering users in learning how to manage cravings, change dysfunctional thinking, developing refusall skills, adaptive coping to problems, understanding pros and cons of use and address distortions in thinking, counteracting helplessness and hopelessness, obtaining positive social support and developing a healthy lifestyle. CBT strategies are developed in Modules 3 to 6 to that focuses on drug recovery skills. Module 3 develops behavioral strategies to cope with cravings, Modules 4 and 5 focuses on strategies to managing external triggers and drug refusal skills, and Module 6 hones strategies to develop a healthy lifestyle. CBT strategies are also used in building life skills. Module 7 teaches PWUDs reframing as a means of managing negative emotions that may trigger their use. 
Module 8 hones participants interpersonal skills, Module 9 focused on ways to rebuilding relationships, and Module 10 develops problemsolving skills (PAP, 2017).

Mindfulness is a meditational practice of being nonjudgmentally aware of the present moment (Bishop et al., 2004). There is emerging evidence of the value of mindfulness in relapse prevention (Zgierska et al., 2009) and it has also been found to be effective in enabling resilience among Filipino disaster survivors (Hechanova, Docena, et al., 2018). In KKDK, mindfulness meditation is included as a centering exercise at the start of each module and in helping participants manage cravings and negative emotions.

The family modules of KKDK are based on family systems theory (e.g., Minuchin, 1974) and consists of three sessions. The first and second session aims to help family members reflect on their dynamics and how it may influence drug use and recovery. In the third session, family members are asked to plan on how to better support the recovering user (PAP, 2017).

Beyond the use of evidence-based approaches to substance use, the design of KKDK considered Philippine cultural values and contextual factors. Given low literacy rates, physical and creative activities are used instead of worksheets when possible. The manual is written in Filipino and in simple language. Skills practice and homework is used to reinforce learning and repetition.

Spirituality play a large role in the lives of Filipino recovering users. A study reports that spiritualty functions as social support for recovering users. When they have problems, experience cravings, stress or negative emotions, recovering users turned to prayer or consulting spiritual advisers (Tuliao \& Liwag, 2011). In recognition of the value of spirituality among Filipinos, ecumenical prayers are incorporated in some modules and opportunity for prayers were included in the closing of the modules. The framework for holistic health also includes the element of spirituality.

Another important cultural adaptation is the manner of delivery. The MIOP and UNODC modules focus on individuals. However, KKDK is designed as a small group intervention because of the Philippines' collectivist culture (Church \& Katigbak, 2002) and following the results of studies that show group-based interventions are a good venue of 
healing among Filipinos (Hechanova \& Waelde, 2017).

In addition, recognizing the important role of the family for Filipinos, KKDK includes modules on rebuilding relationships and family modules to help significant others understand the nature of drug use how to provide support during recovery. Each session requires homework that involve interaction with family members to encourage dialogue and connection with family.

\section{Implementation of Community-Based Interventions}

In McKleroy and colleagues' (2006) intervention design process, the development of an intervention is followed by training of facilitators, pilot-testing, and implementation. The effectiveness of any treatment is, to a large extent, dependent on the commitment and ability of community facilitators (Sparer, 1975). UNODC (2015) guidelines suggest that effective community counselors should have basic counseling skills and be empathic, ethical, respectful, and nonjudgmental. A study suggests that biases of community facilitators against drug users may impede their ability to provide the care that recovering users need (Sheridan, Barnard, \& Webster, 2011). At the same time, a study on non-specialists (e.g., teachers, clergy, nurses, social workers) who take on facilitation roles report that challenges include their limited skills and lack of confidence in delivering community mental health interventions (Coppens et al., 2014).

Beyond the nature of treatment and facilitation, a study reports a number of barriers and drivers in the implementation of communitybased interventions. Inadequate funds, resources and equipment, a lack of skilled personnel, technical support, support from local authorities, and non-acceptance by the community are common barriers to implementation (Belizen et al., 2019). However, these barriers are also mitigated by enablers such as motivated local leaders, intersectoral participation, and the use of local resources (Belizen et al., 2019).

\section{Research Problems}

A previous study by Hechanova, Alianan, et al. (2018) documented 
in detail the needs analysis and design of KKDK. This paper builds on that previous study by describing the outcomes of the facilitators' training and the pilot-testing of the intervention. Specifically, it posed the following questions:

1) Can training significantly increase community facilitators' perceived competence, motivation, self-efficacy and commitment to deliver KKDK?

2) Can the KKDK program significantly increase drug recovery, life skills, and psychological well-being and decrease substance use disorder symptoms of participants?

3) What is the relationship of drug recovery skills, life skills, and family support on substance use disorder symptoms and psychological well-being of participants?

4) What are the barriers and enablers in implementing KKDK?

\section{Study Design}

This study utilized a mixed-method design in two phases. In Phase One, a survey measuring perceived competence, motivation, selfefficacy, and commitment of community facilitators was administered before and after the training program. Open-ended questions were also used to elicit reactions and insights. In Phase Two, surveys measuring drug recovery, life skills, substance use disorder (SUD) symptoms, and psychological well-being were administered before and after the pilot implementation of the intervention. Focus group discussions and interviews were used to elicit feedback from participants and facilitators. Finally, field observations by the research team were gathered as input to implementation challenges and enablers.

\section{PHASE ONE: TRAINING OF COMMUNITY FACILITATORS}

\section{Sample}

A total of 111 community facilitators completed the KKDK facilitators' workshop. Participants' age ranged from 19 to 80 years $(M=41$ years, $S D=16)$. Majority (63\%) were female and more than half of the participants were college graduates (60\%). Majority of 
participants were government employees (64\%) and the rest were church volunteers.

\section{Training}

The initial training for community facilitators was a five-day workshop that covered the theoretical foundations of the program, listening skills, motivational interviewing skills, group facilitation, ethics and implementation issues, and self-care. A core part of the training was the use of small group simulations with each participant served as a facilitator for a module. Coaches were assigned to each group and they provided feedback on participants' facilitation skills, fidelity to module design, and ability to facilitate the program.

\section{Measures}

A survey was administered before and after the facilitators' workshop. It contained four scales (e.g., perceived competence, motivation to help, self-efficacy, commitment) using a five-point scale with 1 as lowest and 5 as highest, and open-ended questions.

Perceived competence is the extent to which facilitators feel they have the knowledge and skills necessary to help recovering users. It was measured using four items: "I have adequate knowledge to help recovering users stop using drugs", "I feel I know enough about causes of drug problems to carry out my role when working with drug users", "I think I have the right to ask recovering users about their drug use", "I feel I have the credibility to help recovering users." Internal consistency of this scale was .70.

Motivation to help is the extent to which facilitators want to help recovering users. It was measured using three items: "I want to help recovering users", "I find it meaningful to help recovering user" and "I feel happy when drug users want to stop." Internal consistency of this scale was .73.

Self-efficacy is the extent to which facilitators feel confident that they can facilitate a drug recovery intervention program. It was measured using two items: "How confident are you that you can help a recovering user remain sober?" and "How confident are you that you 
can facilitate the KKDK?" Items used a 5-point scale with o as low and 4 as high. Internal consistency of this scale was .77.

Commitment is the extent to which facilitators are willing to actually be involved in drug recovery support. It was measured using two items: "How committed are you to help recovering users?" and "How committed are you to facilitate the KKDK?" Internal consistency of this scale was .77.

\section{Results}

Participants' ratings on the different components of the workshop revealed high ratings (on a scale of 1 to 4 with 4 as excellent) in relation to the following: facilitators $(m=3.59)$, coaches $(m=3.45)$, facilities $(m=3.55)$, materials $(m=3.53)$.

Beyond the quantitative data, qualitative feedback from the evaluations revolved around the intervention design, use of manual, workshop methodology, and impact of the workshop. In terms of the intervention design, community facilitators appreciated the design of the KKDK (e.g., "modules had clear objectives and focused on specific skills," "modules had good flow"). They also cited the validity of the content (e.g., "the modules are appropriate to PWUDs and their families," "modules really reflect what happens in real life").

Participants also appreciated the ease of use of the manual and how it provided details on activities, talking points, visual aids, worksheets, and handouts (e.g., "the modules are easy to understand and follow"). However, community facilitators also suggested the need to review the language of the manual (e.g., "some Filipino words are too deep or unfamiliar") and have an English version (e.g., "English manual please"). Others struggled with specific modules and wanted more explanation and examples (e.g., "module 7 on reframing was confusing, need more examples").

Community facilitators also appreciated the hands-on approach used in their training. Specifically, they cited the use of simulations (e.g., "we were able to practice facilitating") and size of groups (e.g., "small groups are good"). Participants also appreciated the guidance of facilitators and coaches (e.g., "we were guided well by coaches").

In terms of impact, paired samples t-test revealed a significant 
change in perceived competence, motivation, and commitment. Cohen's $d$ revealed moderate effect size for perceived competence and motivation and a small effect size for commitment (see Table 2).

The qualitative data confirmed the quantitative data. Community facilitators expressed how the workshop changed their knowledge (e.g., "I learned how to better help recovering users") and skills (e.g., "I learned how to listen better", "I learned how to apply some techniques like breathing to myself"). Participants also reported changes in their attitudes towards drug users (e.g., "I was able to put myself in the shoes of drug users," "I understand better why people use drugs", "I realized how each person's life is important"). The training also strengthened their commitment to help drug users recover (e.g., "I am excited to use what I have learned and deliver this intervention in the community. If I can change even one person's life, then it will be worth it").

\section{PHASE TWO: PILOT AND EVALUATION}

The data from the training and pretest of materials were used to revise the KKDK manual. Once finalized, the intervention was pilot-tested with PWUDs assessed as mild-risk users by doctors. The modules were delivered once or twice a week by the community facilitators. Coaches were assigned to observe every session, provide feedback to community facilitators, and gather evaluation data.

\section{Sample}

Initially, there were 98 participants in the pilot group. However, only 46 participants completed all 15 modules. Statistical analysis was performed to check for significant differences among those who completed and did not complete the program. Results revealed no significant differences except for age. Those who did not complete the program were younger $(M=36, S D=9.39)$ compared to those who completed the program $(M=41, S D=10.56)(t=2.42, p<.05)$. Participants were predominantly male (84\%) and married (56\%). Forty percent were regularly employed, $29 \%$ were contractual employees, and $31 \%$ were unemployed. Participants' ages ranged from 18 to 65 ( $M=39$ years). Age at first use ranged from 8 to 55 years ( $M=26$ years). 


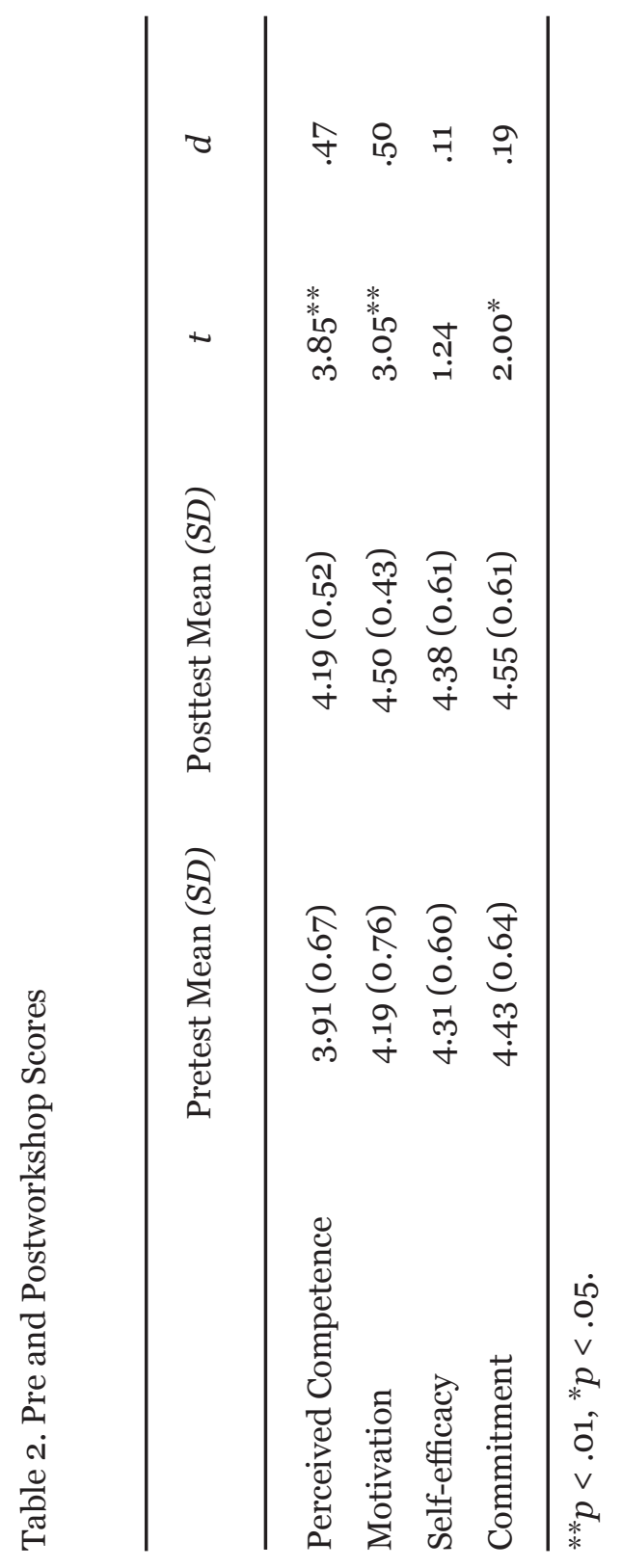


The drug of choice of participants were shabu (methamphetamines) (99\%) and marjiuana (95\%).

\section{Measures}

A survey measuring substance use dependence symptoms, drug recovery skills, life skills, and psychological well-being were administered before and after the program.

Drug recovery skills refers to strategies used to ensure relapse prevention. It was measured using Litman, Stapleton, Oppenheim, Peleg, and Jackson's (1984) Effectiveness of Coping Behaviors Inventory (ECBI). ECBI consists of 36 items describing strategies used in drug recovery such as avoidance, distraction, and cognitive control. Respondents indicated the frequency by which they utilize these behaviors on a 4-point scale ( $3=$ always; $0=$ never). Internal consistency reliability was .92 (pretest) and .94 (posttest).

Life skills refers to abilities to deal with typical problems. Some items were adapted from Sharma's Life Skills (2003) to measure problem solving skills (e.g., "I know the steps to take in solving a problem"), and stress management skills (e.g., "I can identify the source of my stress"). Two items were added to measure relational skills taught in the program (e.g., "I can ask for forgiveness from people I have hurt"). Items were rated on a 5 -point scale (i.e., $5=$ strongly agree; $1=$ strongly disagree) and respondents indicated to which they were able to perform these behaviors. Internal consistency reliability for the pre and posttest was .86 and.89 respectively.

Substanceuse dependencerefersto symptoms of dependencebased on World Health Organization's (WHO) International Classification of Diseases - 10th Edition (ICD-10, 2004). It was measured using the ICD-10 checklist for mental disorders (psychoactive substance use syndromes module) that asks respondents to indicate whether or not they experienced cravings, withdrawal, harmful effects, etc. Internal consistency reliability was .66 (pretest) and .86 (posttest).

Psychological well-being is the presence of positive affect and absence of negative affect. It was measured through the WHO (Five) Well-Being Index (Topp, Østergaard, Søndergaard, \& Bech, 2015). The five items in the scale are associated with positive mood, vitality, and 
general interests, and are positively worded statements. Participants indicate the frequency in which they experiences these using a sixpoint Likert scale: none of the time (0) to all of the time (5). The Cronbach's alpha for the pre and posttest was .85 and .93 respectively.

\section{Focus Group Discussions (FGDs)}

Two FGDs were conducted with participants ( $n=18$ ), and five community facilitators. The FGDs elicited what they appreciated with the intervention as well as what they had difficulty with. It also elicited their suggestions for improving the intervention design.

\section{Procedure}

Ethics approval was obtained for the study from the Ateneo de Manila University. Informed consent was obtained among all participants. In addition, privacy and confidentiality was ensured by making sure that data was collected by researchers directly. The pretest for SUD symptoms and drug recovery skills were administered prior to Module 1, whereas the pretest for life skills was administered prior to Module 7. The posttests were facilitated after Module 12. The scales for family support was administered before and after the family modules.

\section{Data Analysis}

Data was tested for normality and showed that both pre and posttest scores for SUD symptoms were heavily skewed. Logarithmic transformation was conducted on this variable to normalize scores. Paired samples t-test analysis with bootstrapping was used to examine difference in pre and posttest scores.

\section{Results}

Results revealed a significant decrease in substance use dependence symptoms and significant increases in drug recovery skills, life skills, and psychological well-being. Cohen's $d$ was used to 
describe the effect size of the intervention on the various outcomes. As suggested by Cohen (1977), a $d=0.2$ is a small effect size, $d=.50$ is medium, and $d=.80$ is a large effect size. Results revealed a small effect size for life skills and a medium effect size for drug recovery skills and psychological well-being (see Table 3).

\section{Post-program FGDs and Interviews}

The results of the focus group discussions validated the quantitative data and revolved around three themes: the content of the intervention, the methodology or approach, and perceived outcomes. In terms of the content, interviews with community facilitators affirmed the importance of building recovery and life skills: "The program teaches participants how to stop - how to manage their cravings, manage triggers, problem-solve, relate to others - life skills that they can use in everyday life." Others cited specific skills such as managing cravings "I learned how to manage my cravings," "I really learned how to manage my cravings and say 'no'. I have remained sober." Another participant cited improvement in his interpersonal skills, "I learned how to communicate assertively. I used to shout at my kids but I've learned how to communicate with them calmly and they listen."

Facilitators likewise mentioned the methodology and approach of KKDK, particularly the facilitative and interactive nature of the program. As one community facilitator recounted: "In other programs, participants just attend and listen. In KKDK, they are listened to." Another element of KKDK cited by facilitators was the inclusion of family: "In other programs, family members would just bring participants. In KKDK, they are actually involved in planning and providing support”.

In terms of outcomes, participants communicated that there were improvements in their relationship with loved ones and allowed them to reconnect:

My kids avoided me. My parents refused to talk to me. I used to beat up my neighbors, I had so many enemies. I had no friends. Slowly, they saw I was doing everything I could to change. They began talking to me. My kids would come to me. They would tell me that they wished I would not return to the person I used to be. 


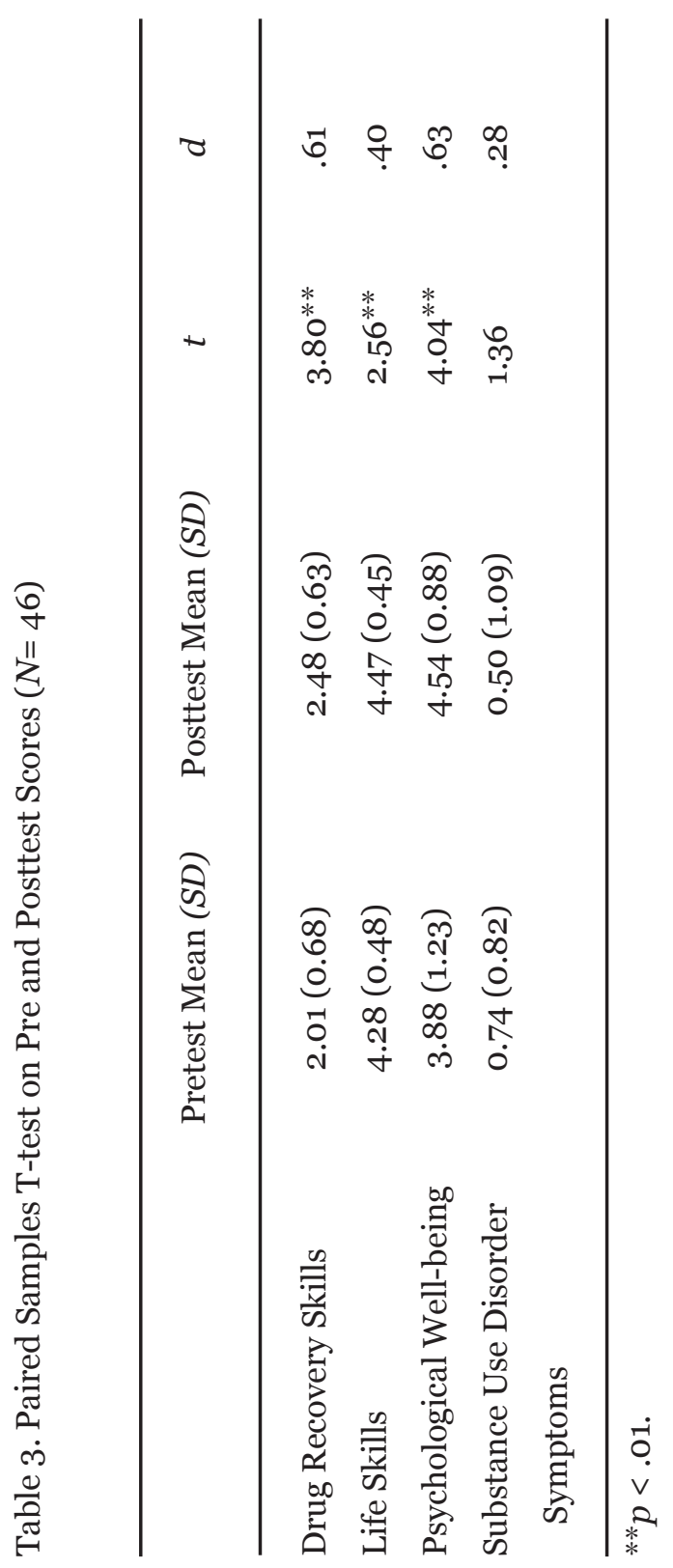


Facilitators also observed that another outcome of the group intervention was the development of peer recovery support. It was observed that after about three to four modules, the groups became cohesive and became important sources of social support. Recounts one facilitator, "They would pick each other up on the way to the session and became a source of support outside the sessions." In one group, one of the participants was sick and the group requested to not continue with the session and visit this participant instead. As one facilitator shared, "They share food, pick each other up. They want to bond and we want to support that. They don't want to leave anyone behind."

\section{Correlational Analysis}

The third research question examined the relationship of recovery skills, life skills, family support, SUD symptoms, and psychological well-being after the program. Correlational analysis revealed that recovery skills is positively correlated with life skills and psychological well-being. Life skills, on the other hand, is negatively associated with SUD symptoms (see Table 4).

\section{Challenges in Implementation}

Despite what appears to be positive outcomes, field observations from coaches revealed a number of challenges in the implementation: (1) bottleneck in screening and assessment, (2) participant attendance, (3) false positives, (4) lack of personnel, (5) lack of skills, (6) stigma, (7) safety and security, (8) drugs in the community, and (9) drug watch lists.

Bottleneck in screening and assessment. Given the requirement that only $\mathrm{DOH}$-certified doctors can conduct assessment and there is a shortage of them, the lack of doctors able to conduct drug dependency evaluation was a major bottleneck. As such, majority of PWUDs remain unassessed and untreated. One PWUD who was awaiting treatment went back to using and was subsequently killed before he could attend the program.

Participant attendance. Another major challenge in the 
Table 4. Correlation of Posttest Scores $(n=46)$

\begin{tabular}{lcccc}
\hline & 1 & 2 & 3 & 4 \\
\hline 1. Recovery Skills & -- & & & \\
2. Life Skills & $.48^{* *}$ & -- & & \\
3. SUD Symptoms & -.19 & $-.34^{*}$ & -- & - \\
4. Psychological Well-being & $.44^{* *}$ & .18 & -.05 & - \\
\hline
\end{tabular}

${ }^{* *} p<.01,{ }^{*} p<.05$.

implementation of the modules was attendance and participant attrition. In some barangays, sessions were held during weekdays, making it difficult for participants who had work to attend. Participants prioritized employment more than attending the sessions thus, those who became employed tended to drop out of the sessions. Although the LGUs recognized the need to shift sessions to weekends, it was difficult to find community facilitators who were willing to run the sessions on Saturdays or Sundays. For PWUDs who were unemployed, the lack of money for transportation was a common reason for not being able to join the sessions.

False positives. Oplan Tokhang had no specific guidelines on who should be invited to surrender and there were reports of quotas being given on the number of surrenderees. As a result, there appeared to be some false positive participants who had not used drugs in many years. There were a few who claimed they never used and were just included in the list. There were also reports of confusion among community officials on how to treat "reformed" pushers who are not users.

Lack of personnel. Given the large number of surrenderees, a major challenge in the implementation was the lack of personnel to facilitate the program. Because there were no budget and structures for community-based drug recovery, the LGU had limited resources to hire facilitators and had difficulty hiring professionals given low salary levels. Given the volume of clients and the limited number of 
personnel, another challenge was the heavy workload of existing community facilitators. In addition, although church volunteers supplemented the personnel to deliver CBDR, their commitment and attendance were not always consistent.

Lack of skill. Beyond the number of personnel, this was another major constraint. Majority of facilitators did not have behavioral science backgrounds, formal training on counseling, or facilitation skills. Thus, despite the training, some facilitators had the tendency to teach or preach rather than facilitate. A few struggled with mastery of the content and maintaining fidelity to modules. To address the issue of skills, one LGU attempted to hire psychometricians as facilitators for CBDR but had difficulty doing so because there were no qualification standards for this position in the Civil Service Commission.

Stigma. Interactions with community leaders and facilitators revealed the presence of stigma in the community. There were some community leaders who viewed PWUDs as criminals and drug use as a sin. Some viewed the tendency to lapse as inability of users to reform or as a sign of failure of the program. Participants also recounted being treated by neighbors as "pests" and criminals.

Safety and security. Field observations also highlighted the role of safety and security. The fear of extra judicial killings was a real concern among participants and families. The presence of police officers who dropped by the sessions to monitor the program also made participants nervous. One facilitator shared, "In the barangay center, we had no privacy and the police would come and go which made participants uneasy. We moved to the church because it was more private and participants trusted us more because we were from the church." Some participants even asked for ID cards to identify them as KKDK participants and avoid being "harassed" by the local police.

Presence of drug pushers in the community. Yet another threat to the participants' safety was the presence of drug pushers in the community. Participants reported that pushers continued to entice participants and some even offered drugs for free. In addition, the lack of alternative employment or livelihood opportunities made it more difficult for some to stay away from drugs, which was their former source of income. 
Lack of aftercare and wrap-around services. Another major challenge in implementation is the lack of aftercare and wraparound social services after participants completed the treatment program. Many recovering users were mostly concerned about finding employment were did not have job skills, were not confident about getting jobs, or were afraid they would just get discriminated upon.

Drug watch lists. In addition to aftercare, a common concern by participants was how their names can be taken out of the drug watch list after they have completed the requirements. There appeared to be no clear process and inconsistent information on the de-listing process.

\section{Enablers in Implementation}

Beyond the aforementioned challenges, there were factors that appeared to enable recovery such as: (1) community support and presence of volunteers, (2) openness to learn and commitment of community facilitators, (3) good governance and partnership, and (4) complementary interventions.

Community support and presence of volunteers. An important enabler was the support given by the community such as volunteers from the church. In some sites, church volunteers facilitated KKDK, in others, they provided the continuing care for participants who completed KKDK.

Openness to learn and commitment of community facilitators. Another critical enabler was the openness and commitment of community facilitators especially given the lack of or low compensation. Even if many did not have technical skills in facilitation, those who were open to coaching showed much improvement in terms of comfort with modules and facilitation skills.

Good governance and partnerships. Good governance was also critical to effective implementation. In particular, a strong relationship between the barangay, church, and police was important to ensure an environment that promoted recovery. In some communities, the barangay captains were very supportive in providing funds for food and materials and ensured a venue for the programs. Some community officials or kagawads were active in conducting home 
visits to invite and ensure continuing attendance of the participants to the program. However, there were also barangays where officials were only peripherally involved and it was mostly church volunteers who implemented the program. Barangays with better support had participants attending more regularly with more favorable outcomes.

Complementary interventions. Another factor that appears to be useful to recovery was the presence of other complementary interventions such as physical exercise, spiritual programs, livelihood, and employment provided by the barangay. As one facilitator commented, "It is not enough that we treat them. We also need to show them we trust them and provide them employment opportunities."

\section{DISCUSSION}

The pilot results, albeit preliminary, suggest that KKDK has potential as an intervention for mild-risk users undergoing community-based drug treatment. However, because the effectiveness of CBDR heavily relies on the quality of facilitation, the manner in which facilitators are trained is crucial. The training of community facilitators requires a hands-on approach and simulation of the modules. Coaching supports module fidelity and provides feedback to enhance participants' facilitation skills. This approach appears to increase facilitators perceived competence and motivation.

However, field observations suggest the importance of sustained coaching and monitoring of community facilitators. In addition, another study suggested that for health workers, constantly dealing with mental health issues can be quite stressful and may cause compassion fatigue and burnout (Rahman, Malik, Sikander, Roberts, \& Creed, 2008). Thus, beyond training for technical skills, supervisory mechanisms may also need to incorporate debriefing sessions, selfcare, and encourage peer support towards the well-being of community facilitators (Rahman et al., 2008).

The evaluation of the pilot implementation of KKDK revealed that the program enabled significant improvements in drug recovery skills. Results also suggest that drug recovery skills are associated with increased psychological well-being. The qualitative results also highlighted the importance of helping users manage their cravings 
and external triggers. This affirms studies suggesting that the most successful treatment interventions enhance coping, reduce cravings, manage triggers, and prevent relapse (Skewes \& Gonzalez, 2013).

Both quantitative and qualitative results highlighted the value of the intervention in improving life skills, such as interpersonal skills, managing emotions, and problem solving. This reinforces Skewes and Gonzalez's (2013) conclusion that improving life skills enhances social support for sobriety and helps establish a lifestyle free of substance use that is critical to long-term recovery.

In terms of intervention design, community facilitators affirmed the validity of the design and the approach used. The results support the evidence in other countries on the effectiveness of motivational interviewing (Miller \& Rollnick, 2012), cognitive behavior therapy (Magill \& Ray, 2009), and mindfulness (Li, Howard, Garland, McGovern, \& Lazar, 2017) in helping recovering drug users.

The results likewise highlight the value of adapting interventions to suit a collectivist culture. In particular, the use of creative, physical and interactive methodologies appears to work well for Filipino participants. An important adaptation of KKDK was the use of groups. The group format is practical as it addresses the lack of mental health resources and enables helping as many clients as possible. Beyond this, the bond that develops within the groups is especially important in providing social support. Results validate previous findings that group interventions are a good venue of healing among Filipinos (Hechanova, Waelde, \& Ramos, 2016). As explained by Engelbrecht and Jobson (2016), small groups can reduce shame, isolation, and helplessness. In addition, small groups also facilitated the development of peer recovery groups. Given the high possibility of relapse, studies emphasize the importance of continuing care or peer recovery groups (Hennessey \& Fisher, 2015).

Moreover, a key cultural adaptation of KKDK was the engagement of family members through the homework and the family modules. Qualitative results highlighted the importance of strengthening family support which is consistent with literature on the important role of family in recovery of users (Duffy \& Baldwin, 2013).

However, the difficulties of participants particularly in cognitive reframing deserves some reflection. The findings are consistent with 
a study among Filipino disaster survivors that reports participants' difficulty in parsing thoughts and emotions (Hechanova et al., 2015). However, this is not necessarily unique to the Philippines. A study on the use of CBT in China suggests that the Chinese favor behavioral tasks versus cognitive tasks and are uncomfortable with accessing their internal processes because of their value for emotional restraint (Guo \& Hanley, 2015).

The study also highlights the use of participatory approaches in designing community-based interventions. Community-based participatory action research (CBPR) ensures the cultural and contextual validity of programs. The involvement of community stakeholders also improves relationships between researchers and community members and builds the capacity of communities to implement and sustain interventions (Collins et al., 2018).

In terms of barriers and drivers to the implementation, results reveal a number of factors that are similar to those found in other developing countries. Challenges such as the lack of funding, lack of skilled personnel, heavy workload, inadequate equipment and resources, lack of technical support, and lack of support from local authorities have also been cited in studies in other countries (Belizen et al., 2019; Long et al., 2018). The lack of integration with the overall health system was also cited in a study on community-based interventions in China (Long et al., 2018). At the same time, enablers such as motivated local leaders and intersectoral participation are similarly cited in other studies (Belizen et al., 2019). However, what appears to be unique in the case of CBDR in the Philippines is the emergence of safety and security issues which may be a function of the generally punitive approach to drug use in the country.

\section{Limitations}

An important limitation of the pilot study is its design. The pilot utilized a small sample size with no randomization and absence of control groups. The sample size was related to the bottleneck in screening. Randomization was also not feasible given the bottleneck in assessment, the presence of drug-related killings, and the pressure from community leaders to provide the CBDR intervention. Future 
research using true experimental designs is important to provide more robust evidence on the effectiveness of the KKDK.

Future studies may also wish to explore possible outcomes including self-efficacy, readiness to change, and actual substance use through biological markers. In additional, posttest surveys were only done immediately after the program. Longitudinal studies are important to examine the extent to which the intervention is able to ensure long-term recovery.

\section{Implications for Practice}

The pilot evaluation of the KKDK suggests it has promise as a CBDR intervention for mild-risk drug users. However, it must be noted that the program was designed based on a needs analysis of urban poor PWUDs in Metro Manila. Further customization may be required depending on location and presence of subcultures in the Philippines. Translations of KKDK to various dialects as well as tweaking the nature of metaphors, symbols, and approaches may also be needed to ensure relevance to various contexts. In addition, given the complexity of some modules, having booster sessions may be considered in the aftercare programs.

Bottlenecks in the current system preventing recovering users from accessing treatment need to be addressed. Constraints in the participation of recovering users suggest the need to provide drug treatment when it is accessible to clients such as during weekends so it does not conflict with livelihood. In addition, the continued participation of clients may be addressed by providing support for transportation or providing incentives (e.g., food, groceries) for attendance.

Given the dearth in manpower and financial resources, results suggest the potential of tapping church and civil society organizations to deliver community-based interventions. However, the lack of accountability of volunteers is also a barrier to the implementation of the program. In addition, given attrition problems, there is a need for flexibility on the part of the community and its facilitators to deliver the intervention during weekends when participants are most available. When available, tapping facilitators with behavioral science 
backgrounds (nurses, psychometricians, psychologists, counselors, social workers, and midwives) or prior training and experience in group facilitation would be ideal.

Observations on the need to improve the consistency and quality of facilitation suggest that psychologists who work in communities need to take on roles such as trainers and coaches and need to have presentation, facilitation, and coaching skills (Martino et al., 2010). As interventionists, community psychologists require skills in needs analysis, intervention design, project and change management, and evaluation (Wight, Wimbus, Jepson, \& Doi, 2015). As change agents, community psychologists need to have an array of nontechnical skills including an ecological and systems perspective as well as long-term thinking, change management, and collaborative skills (Wolff, 2014). All these aforementioned roles and competencies require a different way of training psychologists. In fact, Kelly (1970) recommends that university education is best supplemented by field training, exposure, and long-term partnering with communities.

Field observations on the challenges in implementation support the perspective that drug use is a complex health condition that has social, psychological, and biological dimensions (UNODC, 2014). UNODC (2008) describes five types of recovery capital: human capital (good health, knowledge, and skills), physical and financial assets (economic and financial assets), natural capital (resources for livelihood), social capital (social support and safety net). Even if the program increases the recovery and life skills of individuals, a critical issue surrounds the question of providing the other recovery capitals that participants need to achieve quality of life.

A lack of family and community support, prospects for employment, and continued aftercare may only frustrate recovering users and make them fall back into old patterns. In addition, group interventions such as KKDK may also be inadequate for individuals with serious problems who may require counseling or therapy. Furthermore, a climate of fear and punishment may prevent recovering users from seeking help, hence the importance of addressing stigma in both community and national levels.

The implementation challenges suggest that treatment cannot occur in a vacuum and the link between poverty and its consequences 
requires holistic approaches to the issue of drug use. Thus, psychosocial treatments such as KKDK cannot stand alone without complementary interventions related to employment, physical health, spiritual guidance, educational enrichment, and the like. In addition, ensuring that communities have the resources and structures to implement CBDR is critical in ensuring its effective implementation and sustainability.

\section{AUTHORS' NOTES}

This research was funded by Commission on Higher Education under its K to12 DARE TO Grant-in-Aid program. The study was also done in partnership with the Quezon City government and the Diocese of Novaliches.

\section{REFERENCES}

Beck, A. T., Wright, F. D., Newman, C. F., \& Liese, B. S. (1993). Cognitive therapy of substance abuse. New York: Guilford Press.

Belizen, M., Chaparro, R., Santero, M., Elorriaga, N., Kartschmit, N., Rubenstein, A., \& Irazola, V. (2019). Barriers and facilitators for the implementation and evaluation of community-based interventions to promote physical activity and healthy diet: A mixed methods study in Argentina. International Journal of Environmental Research and Public Health, 16, 213-227.

Bishop, S. R., Lau, M., Shapiro, S., Carlson, L., Anderson, N. D., Carmody, J., . . . Devins, G. (2004). Mindfulness: A proposed operational definition. Clinical Psychology: Science and Practice, 11, 230-241. doi:10.1093/clipsy.bpho77

Cepeda, M. (2016, September 22). DDB wants law institutionalizing community-based drug treatment. Retrieved from https:// www.rappler.com/nation/147020-ddb-law-institutionalizecommunity-based-drug-rehabilitation

Church, A., \& Katigbak, M. S. (2002). Studying personality traits across cultures: Philippine examples. Online Readings in Psychology and Culture, 4(4). doi:10.9707/2307-0919.1039 
Cohen, J. (1977). Statistical power analysis for the behavioral sciences. London, UK: Routledge.

Collins, S. E., Clifasefi, S. L., Stanton, J., The LEAP Advisory Board, Straits, K. J. E., Gil-Kashiwabara, E., . . Wallerstein, N. (2018, January 22). Community-based participatory research (CBPR): Towards equitable involvement of community in psychology research. American Psychologist. Advance online publication. http://dx.doi.org/10.1037/ampoooo167

Coppens, E., Van Auenhove, C., Iddi, S., Arensman, E., Gottlebe, K., Koburger, N., . . Hegerl, U. (2014). Effectiveness of community facilitator training in improving knowledge, attitudes, and confidence in relation to depression and suicidal behavior. Results of the OSPI-Europe intervention in four European countries. Journal of Affective Disorders, 165, 142-150.

Duffy, P., \& Baldwin, H. (2013). Recovery post treatment: Plans, barriers and motivators. Substance Abuse Treatment, Prevention, and Policy, 8(6), 1-12.

Engelbrecht, A., \& Jobson, L. (2016). Exploring trauma associated appraisals in trauma survivors from collectivistic cultures. SpringerPlus, 5(1), 1-11. doi:10.1186/s40064-016-3043-2

Guo, F., \& Hanley, T. (2015). Adapting cognitive behavioral therapy to meet the needs of Chinese clients: Opportunities and Challenges. PsyCh Journal 4, 55-65. doi:10.1002/pchj.75

Hechanova, M. R., Alianan, A., Calleja, M., Melgar, I., Acosta, A., Villasanta, A., . . . Cue, M. (2018). The development of a community-based drug intervention for Filipino drug users. Journal of Pacific Rim Psychology, 12(12), 1-10. doi:10.1017/ prp.2017.23

Hechanova, M. R., Docena, P., Alampay, L., Acosta, A., Porio, E., Melgar, I., \& Berger, R. (2018). Evaluation of a resilience intervention for Filipino displaced survivors of Super Typhoon Haiyan. Disaster Prevention and Management, 27, 346-359

Hechanova, M. R., \& Waelde, L. C. (2017). The influence of culture on disaster mental health and psychosocial interventions in Southeast Asia. Mental Health, Religion and Culture, 2O(1), 3144.

Hechanova M. R., Waelde, L. C., Docena, P., Alampay, L. P., Alianan, 
A., Flores, J., Ramos, P. A., \& Melgar, I. (2015). The development of Katatagan: A resilience intervention for Filipino disaster survivors. Philippine Journal of Psychology, 48(2), 105-131.

Hechanova, M. R., Waelde, L. C., \& Ramos, P. A. (2016). Evaluation of a group-based resilience program for Typhoon Haiyan survivors. Journal of Pacific Rim Psychology, 10(12), 1-10.

Hennessey, E. A., \& Fisher, B.W. (2015). A meta-analysis exploring the relationship between 12-step attendance and adolescent substance use relapse. Journal of Groups in Addiction Recovery, 10, 79-96.

Hodge, D., Jackson, K., \& Vaugh, M. (2012). Culturally-sensitive interventions and substance use: A meta-analytic review of outcomes among minority youth. Social Work Research, 36, 1119.

Kelly, J. G. (1970). Antidote for Arrogance. American Psychologist 25(6), 524-531.

Li, W., Howard, M., Garland, E., McGovern, P., \& Lazar, M. (2017). Mindfulness treatment for substance misuse: A systematic review and meta-analysis. Journal of Substance Abuse Treatment, 75, 62-96.

Litman, G. K., Stapleton, J., Oppenheim, A. N., Peleg, M., \& Jackson, P. (1984). The relationship between coping behaviors, their effectiveness and alcoholism. British Journal of Addiction, 79, 283-291.

Lukoff, I. (1980). Towards a sociology of drug use. In D. J. Lettieri, M. Sayers, \& H. Waalenstein (Eds.), Theories of drug abuse: Selected contemporary perspectives (pp. 201-211). Maryland, USA: National Institute on Drug Abuse.

Long, H., Huang, W., Zheng, P., Li, J., Tao, S. Tang, S., \& Abdullah, A. (2018). Barriers and facilitators of engaging community health workers in non-communicable disease (NCD) prevention and control in China: A systematic review (2006-2016). International Journal of Environmental Research and Public Health, 15, 23782330.

Magill, M., \& Ray, L. (2009). Cognitive-behavioral therapy with adult alcohol and illicit drug users: A meta-analysis of randomized control trials. Journal of Studies on Alcohol and Drugs, 7o(4), 
516-528.

Martino, S., Ball, S., Nich, C., Canning-Ball, M., Rounsaville, B., \& Carroll, K. (2010). Teaching community program clinicians motivational interviewing using expert and train the trainer strategies. Addiction, 106(2), 428-441.

McKleroy, V.S., Gailbraith, J. S., Cummings, B., Jones, P., Harshbarger, C., Collins, C., . . . the ADAPT team. (2006). Adapting evidencebased behavioral interventions for new settings and target populations. AIDS Education and Prevention, 18, 59-73.

Miller, W. R., \& Rollnick, S. (2012). Motivational interviewing: Helping people change (3rd ed.). New York, NY: Guillford Press.

Minuchin, S. (1974). Families and family therapy. Cambridge, MA: Harvard University Press.

Psychological Association of the Philippines. (2017). Katatagan Kontra Droga sa Komunidad: A community-based drug relapse program. QC, Philippines: PAP.

Rahman, A., Malik, A., Sikander, S., Roberts, C., \& Creed, R. (2008). Cognitive behavioral therapy based intervention by community mental health workers for mothers with depression and their infants in rural Pakistan: A cluster-randomized controlled trial. The Lancet, 372, 902-909.

Robles, E., Maynard, B., Salas-Wright, C., \& Todic, J. (2018). Culturally-adapted substance use for Latino Adolescents: A systematic review and meta-analysis. Research on Social Work Practice, 28(7), 789-801.

Sadongdong, M. (2018, February 9). 11,00o names figure in validated drug watch list. Manila Bulletin. Retrieved from https://news. mb.com.ph/2018/02/09/11000-names-figure-in-validateddrug-watch-list/

Sharma, S. (2003). Measuring life skills of adolescents in a secondary school of Kathmandu: An experience. Kathmandu University Medical Journal, 1(3), 170-176

Sheridan, J., Barnard, M., \& Webster, S. (2011). Influences on the provision of drug services in England: The experiences and view of front line treatment workers. Health and Social Care in the Community, 19(4), 403-411.

Skewes, M., \& Gonzalez, V. (2013). The biopsychosocial model of 
addiction. In P. Miller (Ed.), Principles of addiction (Vol. 1, pp. 6170). AcademicPress. doi:10.1016/B978-0-12-398336-7.00006-1.

Sparer, G. (1975). OEO drug treatment programs: Are communitybased, nonprofessional, drug-free programs effective? Public Health Reports (1974-), 9o(5), 455-459. Retrieved from http:// www.jstor.org/stable/4595305

Stanton, M. D. (1980). A family theory of drug abuse. In D. J. Lettieri, M. Sayers, \& H. Waalenstein (Eds.), Theories of drug abuse: Selected contemporary perspectives (pp. 147-156). Maryland, USA: National Institute on Drug Abuse.

Substance Abuse and Mental Health Services Administration (SAMHSA). (2006). Counselor's treatment manual: Matrix intensive outpatient treatment for people with stimulant use disorders. DHHS Publication No. (SMA)07-4152. Rockville, MD: Substance Abuse and Mental Health Services Administration.

Tuliao, A., \& Liwag, E. (2011). Predictors of relapse in Filipino male methamphetamine users: A mixed methods approach. Journal of Ethnicity in Substance Abuse, 10, 162-179.

United Nations Office of Drug and Crime. (2008). Drug dependence treatment: Sustained recovery management. Retrieved from https://www.unodc.org/docs/treatment/111SUSTAINED_ RECOVERY_MANAGEMENT.pdf

United Nations Office of Drug and Crime. (2014). Community based treatment and care for drug use and dependence. Retrieved from http://www.unodc.org/documents/southeastasiaandpacific/ cbtx/cbtx_brief_EN.pdf

Wight, D., Wimbus, E., Jepson, R., \& Doi, L. (2015). Six steps in quality intervention development. Journal of Epidemiology Community Health, o, 1-6. doi:10.1136/jech-2015-205952

Windsor, L. C., Jemal, A., \& Alessi, E. (2015). Cognitive behavioral therapy: A meta-analysis of race and substance use outcomes. Cultural Diversity and Ethnic Minority Psychology, 21(2), 300313 .

World Health Organization (WHO). (2004). The ICD-10 symptoms checklist. Retrieved from https://www.who.int/substance_abuse /research_tools/en/english_icd10.pdf

Wolff, T. (2014). Community psychology practice: Expanding the 
impact of psychology's work. American Psychologist, 69(8), 803813.

Zgierska, A., Rabago, D., Chawla, N., Kushner, K., Koehler, R., \& Marlatt, A. (2009). Mindfulness meditation for substance use disorders: A systematic review. Substance Abuse, 4, 266-294. 